\title{
Modeling, control and analysis of limit cycle walking on slippery road surface
}

\author{
Fumihiko Asano - Yasunori Kikuchi • \\ Masahiro Shibata
}

Received: 29 December 2013 / Revised: 2 March 2014 / Accepted: 5 March 2014 / Published online: 22 March 2014

(C) Springer-Verlag Berlin Heidelberg 2014

\begin{abstract}
This paper investigates the feasibility and fundamental gait properties of active and passive limit-cycle walkers that walk on slippery road surface. First, we introduce the model of an underactuated spoked walker with a torso and mathematically analyze the collision model on the assumption of sliding contact with the ground to identify the condition for achieving instantaneous stance-leg exchange. Second, we develop the equation of motion incorporating sliding friction acting on the stance-leg-end and numerically show that the walker can generate a stable walking gait by applying a simple control of the torso. Furthermore, we discuss the case of passive dynamic walking on slippery slope using the same model but without considering the torso dynamics.
\end{abstract}

Keywords Limit cycle walking - Gait generation - Rimless wheel $\cdot$ Sliding contact

\section{Introduction}

In modeling of limit cycle walking including passive dynamic walking [1], it is commonly assumed that the end point of the stance leg is always in contact with the ground without sliding [2,3]. This implicitly supports that the contact point develops sufficient friction during motion and at impact. On slippery road surface, however, this assumption cannot hold

F. Asano $(\bowtie) \cdot$ Y. Kikuchi $\cdot$ M. Shibata

School of Information Science, Japan Advanced Institute of Science and Technology, 1-1 Asahidai, Nomi, Ishikawa 923-1292, Japan

e-mail: fasano@jaist.ac.jp

Y. Kikuchi

e-mail: yasunori_kikuchi@jaist.ac.jp

M. Shibata

e-mail: masahiro.shibata@jaist.ac.jp and the robot equation must be reconsidered taking the sliding contact into account. This issue should not be ignored in development of walkers that are highly adaptive to unknown environment but there are few studies on it.

It is not difficult to develop the model of a limit-cycle walker that moves sliding on the floor, but the collision with the slippery floor must be carefully modeled according to the condition. The problem of how to determine the motion immediately after impact has been investigated in several studies. Bourgeot et al. [4] discussed the variety of the postimpact motion of a dynamic walker that falls down as a 1DOF rigid body based on the concept of rocking block, and they divided the possible motion into four cases according to the relation between the pre-impact velocity and the postimpact one. Font-Llangunes and Kövecses [5] also discussed this issue and analyzed the post-impact state of a compasslike biped robot. They identified the condition for transition to double-limb support (DLS) based on the vertical velocity of the end-position of the rear leg. On the slippery floor, however, this issue has not been discussed in detail until now.

Based on the observations, this paper addresses two issues; one is identifying the condition for achieving instantaneous exchange of the stance leg while sliding, and the other is generation of stable walking gaits on a slippery road surface. First, we introduce the model of an underactuated spoked walker with a torso that falls down as a 1-DOF rigid body immediately before impact [6], and develop the inelastic collision model on the assumption that the walker is sliding at impact (during the collision phase). We then identify the condition that the rear leg leaves the ground immediately after landing of the fore leg. We analytically derive the impulse vector which is derived as a zero-time integral of the impulsive forces at impact and examine the sign of each element to determine unilateral constraint $[7,8]$. Second, we develop the equation of motion incorporating sliding friction acting 
on the stance-leg-end and numerically test the feasibility of stable limit cycle walking on a slippery road surface. The sliding friction is mathematically developed as the simplest model that represents the Coulomb's friction law but without considering the static friction effect; it corresponds to the condition of frozen or oily road surface. Through numerical analysis of the gait properties, we show that there is an optimal friction coefficient for achieving the most efficient walking gait. Furthermore, we extend the analysis to the case of passive dynamic walking by using the same model but without controlling the torso.

The subsequent sections are organized as follows. Section 2 develops the collision model and derives the condition for achieving instantaneous stance-leg exchange. Section 3 describes the equation of motion and output following control for generating a level gait. Section 4 analyzes the gait efficiencies and properties through numerical simulations. Section 5 discusses passive dynamic walking on slippery slope. Finally, Sect. 6 concludes this paper and describes the future research direction.

\section{Collision analysis}

\subsection{Underactuated spoked walker with torso}

Figure 1 shows the model of an underactuated spoked walker which is composed of a twelve-legged rimless wheel (RW) $[2,3]$ and a torso. Let $m_{1}(\mathrm{~kg})$ and $I_{1}\left(\mathrm{~kg} \mathrm{~m}^{2}\right)$ be the mass and inertia moment of the RW. Let $m_{2}(\mathrm{~kg})$ and $I_{2}\left(\mathrm{~kg} \mathrm{~m}^{2}\right)$ be those of the torso. The leg length or the radius of the RW is $l(\mathrm{~m})$. The length of the torso is $2 r(\mathrm{~m})$ and this is connected to the RW at the central joint. This walker can exert a joint torque, $u(\mathrm{Nm})$, between the stance leg and the torso. The torso functions as a reaction wheel for the RW; the stance leg can use the reaction torque for propulsion [6].

\subsection{Inelastic collision model}

Let $\boldsymbol{q}=\left[\begin{array}{llll}x & z & \theta_{1} & \theta_{2}\end{array}\right]^{\mathrm{T}}$ be the generalized coordinate vector. Here, $(x, z)$ is the position of the stance-leg end, $\theta_{1}$ is the stance-leg angle with respect to vertical, and $\theta_{2}$ is the torso angle with respect to horizontal. The inelastic collision of the fore leg (the next stance leg) with the ground is then modeled as

$\boldsymbol{M}(\boldsymbol{q}) \dot{\boldsymbol{q}}^{+}=\boldsymbol{M}(\boldsymbol{q}) \dot{\boldsymbol{q}}^{-}+\boldsymbol{J}_{I}(\boldsymbol{q})^{\mathrm{T}} \lambda_{I}$,

where the superscripts "-" and "+" stand for immediately before and immediately after impact. Note that $\boldsymbol{q}$ in Eq. (1) is equal to $\boldsymbol{q}^{-}=\boldsymbol{q}^{+} . \lambda_{I}$ is the impulse vector given as the zerotime integral of the impulsive force at impact. $\boldsymbol{M}(\boldsymbol{q}) \in \mathbb{R}^{4 \times 4}$ is the inertia matrix and is detailed as

$\boldsymbol{M}(\boldsymbol{q})=\left[\begin{array}{cccc}m_{t} & 0 & m_{t} l \cos \theta_{1} & 0 \\ 0 & m_{t} & -m_{t} l \sin \theta_{1} & 0 \\ m_{t} l \cos \theta_{1} & -m_{t} l \sin \theta_{1} & m_{t} l^{2}+I_{1} & 0 \\ 0 & 0 & 0 & I_{2}\end{array}\right]$,

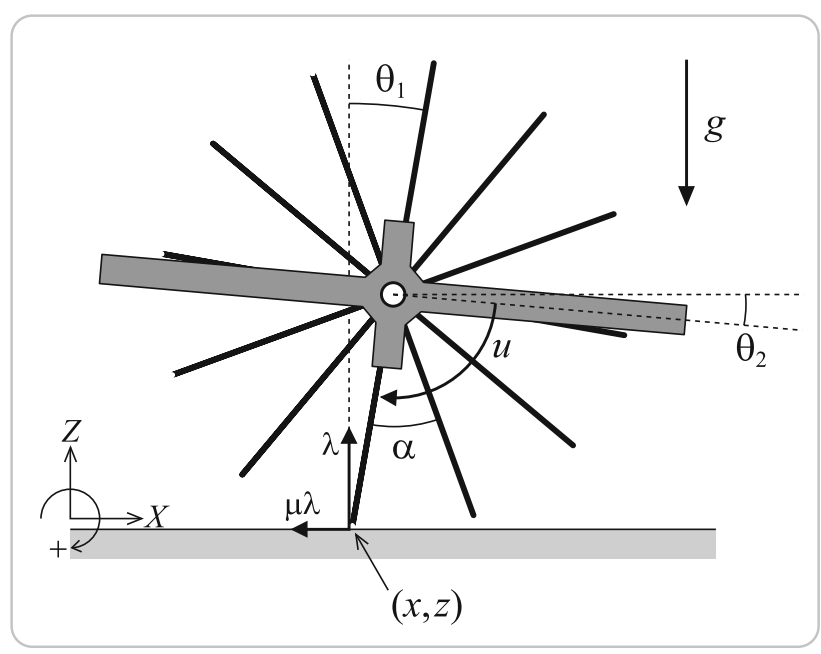

Fig. 1 Model of underactuated rimless wheel with torso

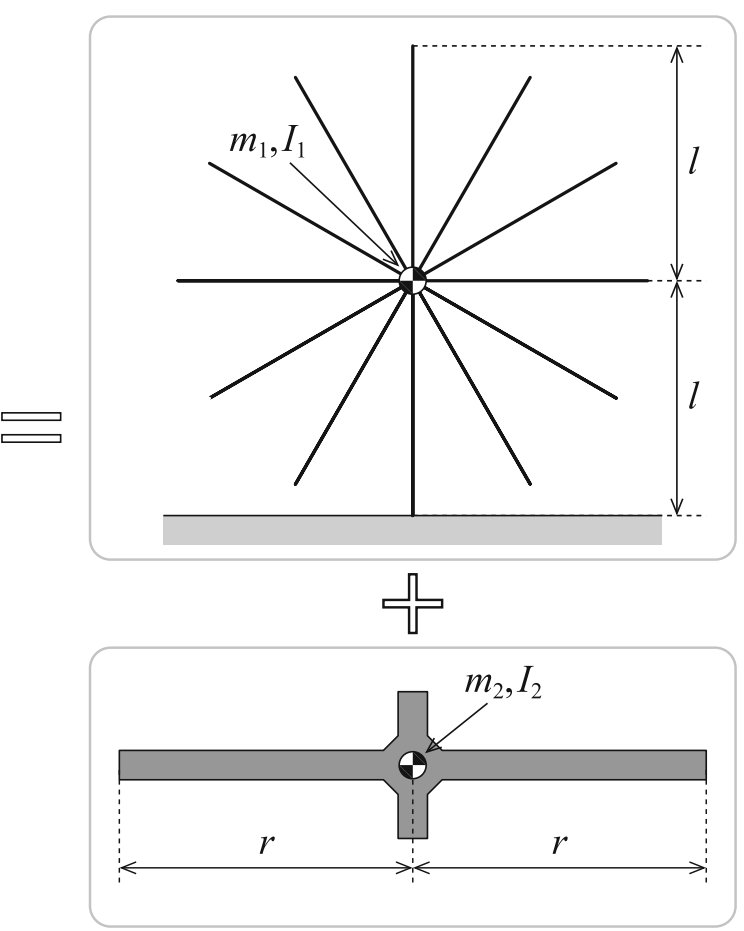


where $m_{t}:=m_{1}+m_{2}[\mathrm{~kg}]$ is the total mass. The size of the Jacobian matrix, $\boldsymbol{J}_{I}(\boldsymbol{q})$, is non-unique and changes according to the condition for velocity constraint at impact. In the following, we describe the conditions in detail.

\subsection{Condition for instantaneous stance-leg exchange}

First, we analytically derive the condition for achieving instantaneous stance-leg exchange by assuming that the motion transitions to DLS after impact.

If we assume that the fore leg slides at impact, that is, the end-point is not constrained in the $X$-direction, the only velocity constraint condition is given by

$\frac{\mathrm{d}}{\mathrm{d} t}\left(z+l \cos \theta_{1}^{-}-l \cos \left(\alpha-\theta_{1}^{-}\right)\right)^{+}=0$,

where $\theta_{1}^{-}=\alpha / 2(\mathrm{rad})$.

We generally assume that the contact point between the stance-leg end and the hard ground develops sufficient friction and that the end point does not slide steadily or the constraint condition of $\dot{x}^{+}=0$ always holds. In this case, the walking motion consists of single-limb support (SLS) and instantaneous DLS for stance-leg exchange. On the ice, however, this is not true and we must develop the Jacobian matrix, $\boldsymbol{J}_{I}(\boldsymbol{q})$, in Eq. (1) accordingly. The lack of $\dot{x}^{+}=0$ implies that a redundant DOF is created. We therefore have to concern about the possible emergence of DLS motion after impact $[7,8]$. Transition to DLS motion implies that the walker continues sliding without rotating. In the following, we discuss the problem of how to determine the post-impact motion, SLS or DLS, through mathematical analysis of the impulse.

If we assume that the rear leg does not leave the ground immediately after landing of the fore leg or maintains contact with the ground, the following condition must hold.

$\dot{z}^{+}=0$

In addition, in this paper we assume that the torso is mechanically locked to the RW at impact. This means that the walker falls down as a 1-DOF rigid body immediately before impact.

This velocity constraint condition is then specified as

$\dot{\theta}_{1}^{+}-\dot{\theta}_{2}^{+}=0$.

By summarizing Eqs. (3), (4) and (5), we get

$$
\begin{aligned}
& \boldsymbol{J}_{I}(\boldsymbol{q}) \dot{\boldsymbol{q}}^{+}=\mathbf{0}_{3 \times 1}, \\
& \boldsymbol{J}_{I}(\boldsymbol{q})=\left[\begin{array}{llll}
0 & 1 & -l \sin \theta_{1}^{-}+l \sin \left(\theta_{1}^{-}-\alpha\right) & 0 \\
0 & 1 & 0 & 0 \\
0 & 0 & 1 & -1
\end{array}\right] .
\end{aligned}
$$

Following Eqs. (1) and (6), the impulse vector, $\lambda_{I} \in \mathbb{R}^{3}$, can be solved as
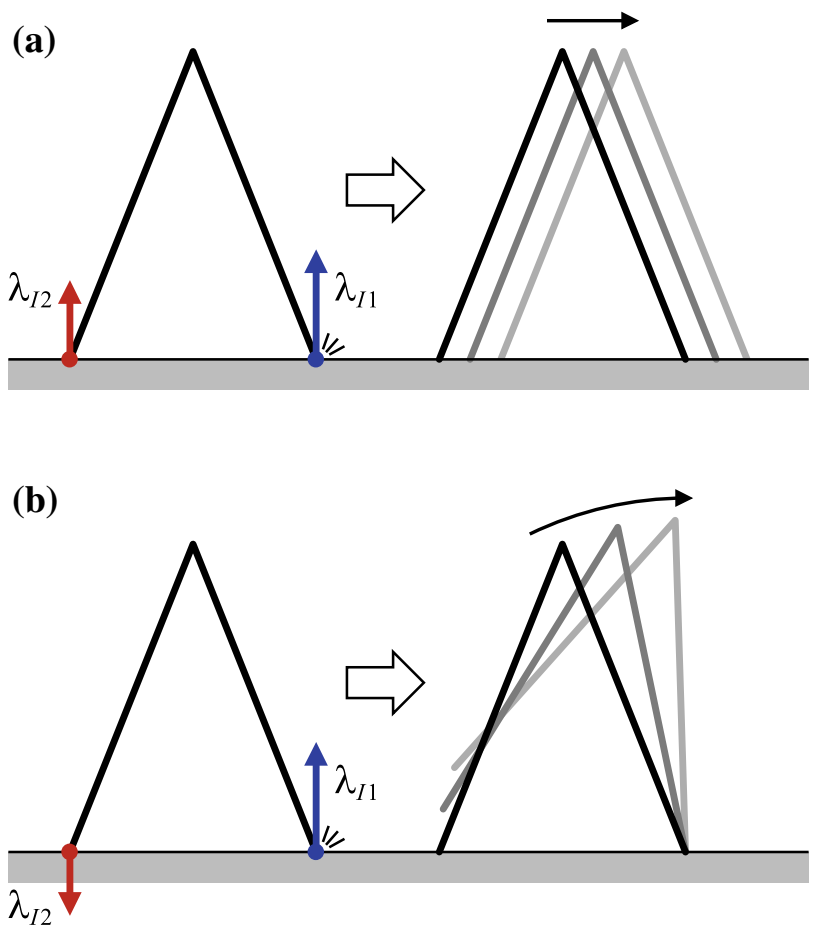

Fig. 2 Model of underactuated rimless wheel with torso

$$
\begin{aligned}
\lambda_{I} & =-\boldsymbol{X}_{I}(\boldsymbol{q})^{-1} \boldsymbol{J}_{I}(\boldsymbol{q}) \dot{\boldsymbol{q}}^{-} \\
& =\left[\begin{array}{c}
\frac{2 I_{t}+m_{t} l^{2}(1-\cos \alpha)}{4 l \sin \frac{\alpha}{2}} \\
-\frac{2 I_{t}-m_{t} l^{2}(1-\cos \alpha)}{4 l \sin \frac{\alpha}{2}} \\
I_{2}
\end{array}\right] \dot{\theta}_{1}^{-}=:\left[\begin{array}{c}
\lambda_{I 1} \\
\lambda_{I 2} \\
\lambda_{I 3}
\end{array}\right],
\end{aligned}
$$

where $\boldsymbol{X}_{I}(\boldsymbol{q}):=\boldsymbol{J}_{I}(\boldsymbol{q}) \boldsymbol{M}(\boldsymbol{q})^{-1} \boldsymbol{J}_{I}(\boldsymbol{q})^{\mathrm{T}}$ and $I_{t}:=I_{1}+I_{2}$ $\left[\mathrm{kg} \mathrm{m}^{2}\right]$ is the total inertia moment. By substituting $\lambda_{I}$ of Eq. (8) into Eq. (1), the velocity vector immediately after impact, $\dot{\boldsymbol{q}}^{+}$, is derived as

$$
\begin{aligned}
\dot{\boldsymbol{q}}^{+} & =\left(\boldsymbol{I}_{4}-\boldsymbol{M}(\boldsymbol{q})^{-1} \boldsymbol{J}_{I}(\boldsymbol{q})^{\mathrm{T}} \boldsymbol{X}_{I}(\boldsymbol{q})^{-1} \boldsymbol{J}_{I}(\boldsymbol{q})\right) \dot{\boldsymbol{q}}^{-} \\
& =\left[\begin{array}{c}
\dot{x}^{-}+l \dot{\theta}_{1}^{-} \cos \frac{\alpha}{2} \\
0 \\
0 \\
0
\end{array}\right]
\end{aligned}
$$

The result of $\dot{\theta}_{1}^{+}=\dot{\theta}_{2}^{+}=0$ implies that the walker begins to slide in the $X$-direction maintaining DLS.

The first element in Eq. (8), $\lambda_{I 1}$, represents the impulse (zero-time integral of impulsive force at impact) in $Z$ direction at the end of the fore leg, and the second element, $\lambda_{I 2}$, represents that of the rear leg as shown in Fig. 2. It is obvious that the $\lambda_{I 1}$ is always positive. The $\lambda_{I 2}$, however, becomes positive only if the following inequality holds.

$m_{t} l^{2}(1-\cos \alpha) \geq 2 I_{t}$ 
Table 1 Physical parameters for walking model

\begin{tabular}{ll}
\hline$m_{1}$ & $2.0 \mathrm{~kg}$ \\
$m_{2}$ & $1.0 \mathrm{~kg}$ \\
$l$ & $1.0 \mathrm{~m}$ \\
$r$ & $1.0 \mathrm{~m}$ \\
$\alpha$ & $30^{\circ}$ \\
$I_{1}=m_{1}\left(\frac{l}{2}\right)^{2}$ & $0.5 \mathrm{~kg} \mathrm{~m}^{2}$ \\
$I_{2}=m_{2} r^{2}$ & $1.0 \mathrm{~kg} \mathrm{~m}^{2}$ \\
\hline
\end{tabular}

If Eq. (11) holds, the motion transitions to non-instantaneous DLS motion as illustrated in Fig. 2a.

The left-hand side in Eq. (11) converges to zero as $\alpha \rightarrow 0$. Small $\alpha$ increases the potential of instantaneous stance-leg exchange. Let us consider a numerical example in the following. By choosing the parameters as listed in Table 1, the value of the left-hand side in Eq. (11) becomes 0.402, whereas that of the right-hand side becomes 3.0. We therefore conclude that non-instantaneous DLS motion does not emerge after impact. The right-hand value in Eq. (11) is the total inertia moment, so the value is large implies that the rotational energy immediately before impact is large. The resultant SLS motion after impact in this case is therefore convincing.

If Eq. (11) does not hold, i.e., $\lambda_{I 2}<0$, the rear leg-end should leave the ground immediately after impact. Therefore the condition of Eq. (4) is not necessary and $\boldsymbol{J}_{I}(\boldsymbol{q}) \in \mathbb{R}^{2 \times 3}$ then becomes

$J_{I}(\boldsymbol{q})=\left[\begin{array}{cccc}0 & 1 & -l \sin \theta_{1}^{-}+l \sin \left(\theta_{1}^{-}-\alpha\right) & 0 \\ 0 & 0 & 1 & -1\end{array}\right]$

By using this, $\lambda_{I} \in \mathbb{R}^{2}$ is derived as

$\lambda_{I}=\frac{4 m_{t} l \sin \frac{\alpha}{2} \dot{\theta}_{1}^{-}}{2 I_{t}+m_{t} l^{2}(1-\cos \alpha)}\left[\begin{array}{c}I_{t} \\ I_{2} l \sin \frac{\alpha}{2}\end{array}\right]$.

It is obvious that all the elements of $\lambda_{I}$ are positive if and only if $\dot{\theta}_{1}^{-}>0$. By substituting Eq. (13) into Eq. (9), we can derive $\dot{\boldsymbol{q}}^{+}$and its elements are detailed as follows.

$\dot{x}^{+}=\dot{x}^{-}+\frac{2 m_{t} l^{3} \sin \frac{\alpha}{2} \sin \alpha}{2 I_{t}+m_{t} l^{2}(1-\cos \alpha)} \dot{\theta}_{1}^{-}$

$\dot{z}^{+}=\frac{2 l \sin \frac{\alpha}{2}\left(2 I_{t}-m_{t} l^{2}(1-\cos \alpha)\right)}{2 I_{t}+m_{t} l^{2}(1-\cos \alpha)} \dot{\theta}_{1}^{-}$

$\dot{\theta}_{1}^{+}=\dot{\theta}_{2}^{+}=\frac{2 I_{t}-m_{t} l^{2}(1-\cos \alpha)}{2 I_{t}+m_{t} l^{2}(1-\cos \alpha)} \dot{\theta}_{1}^{-}$

Note that $\dot{x}^{+}$and $\dot{z}^{+}$in the above equations are the velocities at the end-position of the rear leg immediately after impact. Equation (15) implies that $\dot{z}^{+}$becomes positive if the condition of Eq. (11) holds. This means that the following two conditions are equivalent.
(C1) The velocity of the end-position of the rear leg, $\dot{z}^{+}$, is negative.

(C2) The impulse in vertical direction of the rear leg is positive.

(C1) is the condition Bourgeot et al. [4] and Font-Llagunes and Kövecses [5] derived. (C2) is that Asano et al. [7,8] derived.

Note also that $\dot{\theta}_{1}^{+}\left(=\dot{\theta}_{2}^{+}\right)$of Eq. (16) becomes negative if the condition of Eq. (11) holds. This implies that the RW begins to rotate in the counterclockwise direction after impact. Therefore we can understand that the condition for $\dot{\theta}_{1}^{+}<0$ is equivalent to $(\mathrm{C} 1)$.

The transition rules for the positional coordinates are described in the following. At the start of walking, we set the end-position of the stance leg $(x, z)$ to $(0,0)$. At every impact, we reset $x$ to

$x^{+}=x^{-}+2 l \sin \frac{\alpha}{2}$.

It is obvious that $z^{ \pm}=0$ holds. The angular position of the stance leg, $\theta_{1}$, should be reset to

$\theta_{1}^{+}=\theta_{1}^{-}-\alpha=-\frac{\alpha}{2}$.

Also $\theta_{2}^{ \pm}=0$ must hold on the assumption that the output following control is achieved as described later.

\section{Equation of motion and controller design}

\subsection{Equation of motion considering sliding contact}

The equation of motion corresponding to the generalized coordinate vector, $\boldsymbol{q}$, becomes

$\boldsymbol{M}(\boldsymbol{q}) \ddot{\boldsymbol{q}}+\boldsymbol{h}(\boldsymbol{q}, \dot{\boldsymbol{q}})=\boldsymbol{S} u+\boldsymbol{J}^{\mathrm{T}} \lambda+\boldsymbol{J}_{\mu}^{\mathrm{T}} \lambda$,

where the first term of the right-hand side is the control input vector, the second term is the vector of the holonomic constraint force between the stance-leg end and the floor, and the third term is the vector of the sliding friction force. $\lambda \in \mathbb{R}$ is the Lagrange undetermined multiplier that represents the vertical ground reaction force. In the left-hand side, $\boldsymbol{M}(\boldsymbol{q}) \in \mathbb{R}^{4 \times 4}$ is the same as Eq. (2) and the vectors $\boldsymbol{h}(\boldsymbol{q}, \dot{\boldsymbol{q}}) \in \mathbb{R}^{4}$ and $\boldsymbol{S} \in \mathbb{R}^{4}$ are detailed as follows.

$\boldsymbol{h}(\boldsymbol{q}, \dot{\boldsymbol{q}})=\left[\begin{array}{c}-m_{t} l \dot{\theta}_{1}^{2} \sin \theta_{1} \\ m_{t}\left(g-l \dot{\theta}_{1}^{2} \cos \theta_{1}\right) \\ -m_{t} g l \sin \theta_{1} \\ 0\end{array}\right], \quad \boldsymbol{S}=\left[\begin{array}{c}0 \\ 0 \\ 1 \\ -1\end{array}\right]$

As previously mentioned, the stance-leg exchange is instantaneous and the continuous motion is always SLS. The condition for the holonomic constraint during the stance phase is then given by 
$\dot{z}=\boldsymbol{J} \dot{\boldsymbol{q}}=0, \quad \boldsymbol{J}=\left[\begin{array}{llll}0 & 1 & 0 & 0\end{array}\right]$.

By solving Eqs. (19) and (21) for $\lambda$, we get

$$
\begin{aligned}
\lambda & =-\frac{\boldsymbol{J M}(\boldsymbol{q})^{-1}(\boldsymbol{S} u-\boldsymbol{h}(\boldsymbol{q}, \dot{\boldsymbol{q}}))}{\boldsymbol{J} \boldsymbol{M}(\boldsymbol{q})^{-1}\left(\boldsymbol{J}+\boldsymbol{J}_{\mu}\right)^{\mathrm{T}}} \\
& =\frac{2 m_{t}\left(I_{1}\left(g-l \dot{\theta}_{1}^{2} \cos \theta_{1}\right)-u l \sin \theta_{1}\right)}{2 I_{1}+m_{t} l^{2}\left(1-\cos \left(2 \theta_{1}\right)-\mu \sin \left(2 \theta_{1}\right)\right)} .
\end{aligned}
$$

By observing the sign of $\lambda$, we can check the unilateral constraint.

The sliding friction force in sliding contact is given as the proportional value of the vertical ground reaction force, $\mu \lambda(\mathrm{N})$. Here, $\mu$ is the coefficient of sliding friction and this includes the direction of the friction force. The Jacobian, $\boldsymbol{J}_{\mu} \in \mathbb{R}^{4}$, is then determined as

$\boldsymbol{J}_{\mu}=\left[\begin{array}{llll}\mu & 0 & 0 & 0\end{array}\right]$.

The coefficient of sliding friction, $\mu$, should be, for example,

$\mu(\dot{\boldsymbol{q}})=\mu_{0} \operatorname{sign}(\dot{x})$,

where $\mu_{0}$ is a positive constant. There is no standard value of $\mu_{0}$ for frozen ground because the measured value of it changes in accordance with temperature and ice quality $[9,10]$. We then set small values less than 1.0 for simple modeling. For avoiding chattering, we consider an approximation of Eq. (25) around $\dot{x}=0$ as follows.

$\mu(\dot{\boldsymbol{q}})=-\mu_{0} \tanh (c \dot{x})$

where $c$ is a positive constant for adjusting the sharpness of $\mu$ in the neighborhood of $\dot{x}=0$, and in this paper we choose it as 100. Following Eqs. (19) and (21), we can eliminate $\lambda$ in Eq. (19) and arrange it as follows.

$$
\begin{aligned}
& \boldsymbol{M}(\boldsymbol{q}) \ddot{\boldsymbol{q}}=\boldsymbol{Y}(\boldsymbol{q}, \dot{\boldsymbol{q}})(\boldsymbol{S} u-\boldsymbol{h}(\boldsymbol{q}, \dot{\boldsymbol{q}})) \\
& \boldsymbol{Y}(\boldsymbol{q}, \dot{\boldsymbol{q}}):=\boldsymbol{I}_{4}-\hat{\boldsymbol{J}}(\dot{\boldsymbol{q}})^{\mathrm{T}}\left(\boldsymbol{J} \boldsymbol{M}(\boldsymbol{q})^{-1} \hat{\boldsymbol{J}}(\dot{\boldsymbol{q}})^{\mathrm{T}}\right)^{-1} \boldsymbol{J} \boldsymbol{M}(\boldsymbol{q})^{-1} \\
& \hat{\boldsymbol{J}}(\dot{\boldsymbol{q}}):=\boldsymbol{J}+\boldsymbol{J}_{\mu}(\dot{\boldsymbol{q}})
\end{aligned}
$$

\subsection{Input-output linearization and control input}

Next, let us design a controller to make the robot fall down in a fixed posture immediately before every impact. Let

$y:=\theta_{1}-\theta_{2}=\boldsymbol{S}^{\mathrm{T}} \boldsymbol{q}$

be the control output, and we consider a tracking control of $y$ to $y_{\mathrm{d}}(t)$. The second-order derivative of $y$ with respect to time becomes

$$
\ddot{y}=\boldsymbol{S}^{\mathrm{T}} \ddot{\boldsymbol{q}}=\boldsymbol{S}^{\mathrm{T}} \boldsymbol{M}(\boldsymbol{q})^{-1} \boldsymbol{Y}(\boldsymbol{q}, \dot{\boldsymbol{q}})(\boldsymbol{S} u-\boldsymbol{h}(\boldsymbol{q}, \dot{\boldsymbol{q}})) .
$$

Then we can consider the control input for achieving $y \rightarrow$ $y_{\mathrm{d}}(t)$ as follows.

$$
\begin{aligned}
& u=A(\boldsymbol{q}, \dot{\boldsymbol{q}})^{-1}(v(t)+B(\boldsymbol{q}, \dot{\boldsymbol{q}})) \\
& v(t)=\ddot{y}_{\mathrm{d}}(t)+K_{D}\left(\dot{y}_{\mathrm{d}}(t)-\dot{y}\right)+K_{P}\left(y_{\mathrm{d}}(t)-y\right)
\end{aligned}
$$

The scalar functions $A(\boldsymbol{q}, \dot{\boldsymbol{q}})$ and $B(\boldsymbol{q}, \dot{\boldsymbol{q}})$ are defined as

$A(\boldsymbol{q}, \dot{\boldsymbol{q}}):=\boldsymbol{S}^{\mathrm{T}} \boldsymbol{M}(\boldsymbol{q})^{-1} \boldsymbol{Y}(\boldsymbol{q}, \dot{\boldsymbol{q}}) \boldsymbol{S}$,

$B(\boldsymbol{q}, \dot{\boldsymbol{q}}):=\boldsymbol{S}^{\mathrm{T}} \boldsymbol{M}(\boldsymbol{q})^{-1} \boldsymbol{Y}(\boldsymbol{q}, \dot{\boldsymbol{q}}) \boldsymbol{h}(\boldsymbol{q}, \dot{\boldsymbol{q}})$.

$K_{P}$ and $K_{D}$ are PD gains and are positive constants. $y$ is, however, exactly controlled to follow $y_{\mathrm{d}}(t)$ and PD feedback is not necessary because $y^{+}=-\alpha / 2$ and $\dot{y}^{+}=0$ hold. To smoothly control $y$ from $-\alpha / 2$ to $\alpha / 2$ during the stance phases, we introduce $y_{\mathrm{d}}(t)$ as the following 5-order timedependent function.

$y_{\mathrm{d}}(t)= \begin{cases}\frac{6 \alpha}{T_{\text {set }}^{5}} t^{5}-\frac{15 \alpha}{T_{\mathrm{set}}^{4}} t^{4}+\frac{10 \alpha}{T_{\mathrm{set}}^{3}} t^{3}-\frac{\alpha}{2} & \left(0 \leq t<T_{\mathrm{set}}\right) \\ \frac{2}{2} & \left(t \geq T_{\mathrm{set}}\right)\end{cases}$

Here, note that the time variable, $t$, is reset to zero at every impact. $y_{\mathrm{d}}(t)$ satisfies the following boundary conditions.

$$
\begin{aligned}
& y_{\mathrm{d}}\left(0^{+}\right)=-\frac{\alpha}{2}, \quad \dot{y}_{\mathrm{d}}\left(0^{+}\right)=0, \quad \ddot{y}_{\mathrm{d}}\left(0^{+}\right)=0 \\
& y_{\mathrm{d}}\left(T_{\text {set }}\right)=\frac{\alpha}{2}, \quad \dot{y}_{\mathrm{d}}\left(T_{\text {set }}\right)=0, \quad \ddot{y}_{\mathrm{d}}\left(T_{\text {set }}\right)=0
\end{aligned}
$$

We assume that the output following control is always completed by the next impact.

Note that we must know $\boldsymbol{J}_{\mu}(\dot{\boldsymbol{q}})$ to compute the matrix $\boldsymbol{Y}(\boldsymbol{q}, \dot{\boldsymbol{q}})$ in Eq. (28) and the control input of Eq. (31). More specifically, $\mu(\dot{\boldsymbol{q}})$ must be measured accurately in realtime. The model of $\mu(\dot{\boldsymbol{q}})$ for frozen/oily ground is very simple as specified by Eq. (26) and we assume this can be measured in this paper.

\section{Gait analysis}

\subsection{Typical walking gait and stability}

We chose the initial condition as

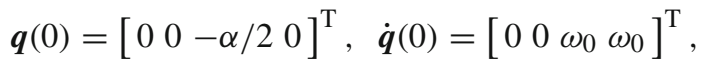

where $\omega_{0}(\mathrm{rad} / \mathrm{s})$ is the initial angular velocity. The walker starts walking from the impact posture shown in Fig. 2 left; this is defined as the 0th impact. The next heel-strike collision is the first impact, and the period between the 0th and the first impacts is called the 0th step. The subsequent impacts and steps are contextually counted.

Figure 3 shows the simulation results of level dynamic walking where $T_{\text {set }}=0.30 \mathrm{~s}, \mu_{0}=0$ and $\omega_{0}=0.80 \mathrm{rad} / \mathrm{s}$. We can see that a stable walking gait is successfully generated on the completely frictionless surface. From Fig. 3a, we can 


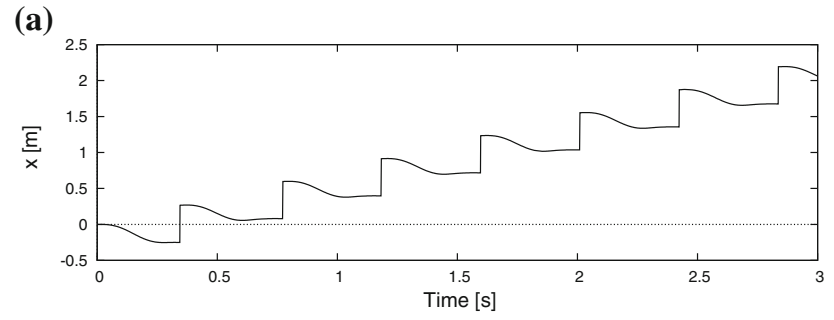

(b)

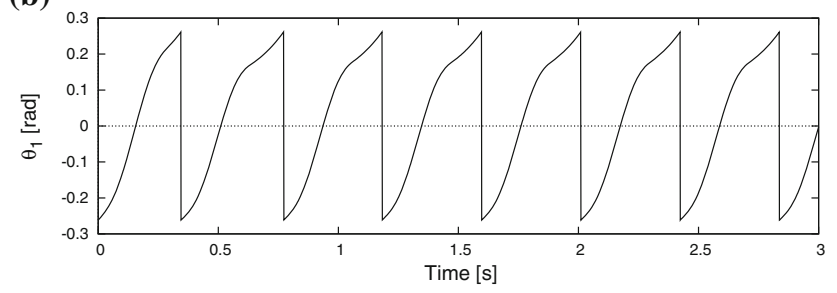

(c)

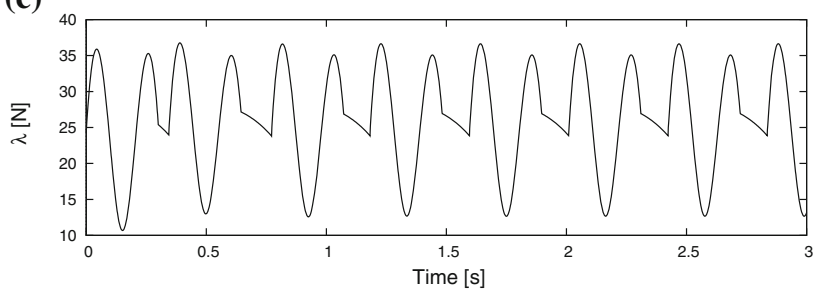

(d)

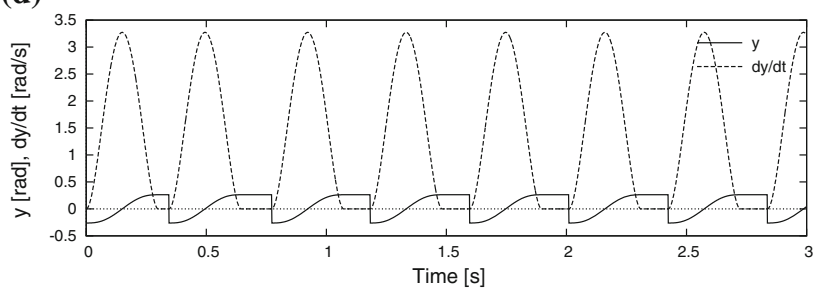

Fig. 3 Simulation results of dynamic walking on slippery surface where $T_{\text {set }}=0.30 \mathrm{~s}$ and $\mu_{0}=0$. a $x$. b $\theta_{1}, \mathbf{c} \lambda \mathbf{d} y$ and $\dot{y}$

also see that $x$ decreases or the contact point slides backward during the stance phases.

Figure 4 shows the evolutions of the gait descriptors for three values of $\omega_{0}$ with respect to the step number. Here, (a) is the step period, and (b) is the walking speed. From Fig. $4 \mathrm{a}$, we can see that the step periods converge to the steady one at a fast convergent rate. From Fig. 4b, however, we can see that the walking speeds are kept different constant values according to the initial angular velocities. We can conclude that the generated gaits are stable but the limit cycles are not unique. The steady walking speed monotonically increases as the initial angular velocity increases.

Figure 5 shows the simulation results of level dynamic walking where $\mu_{0}=0.4$. The initial conditions were chosen as the same in Eq. (33). From the results, we can see that a stable gait is successfully generated. Figure 5a supports that the contact point, $x$, slides forward during the stance phases, that is, the walker in this case can move forward more smoothly than the previous case. The sliding contact with
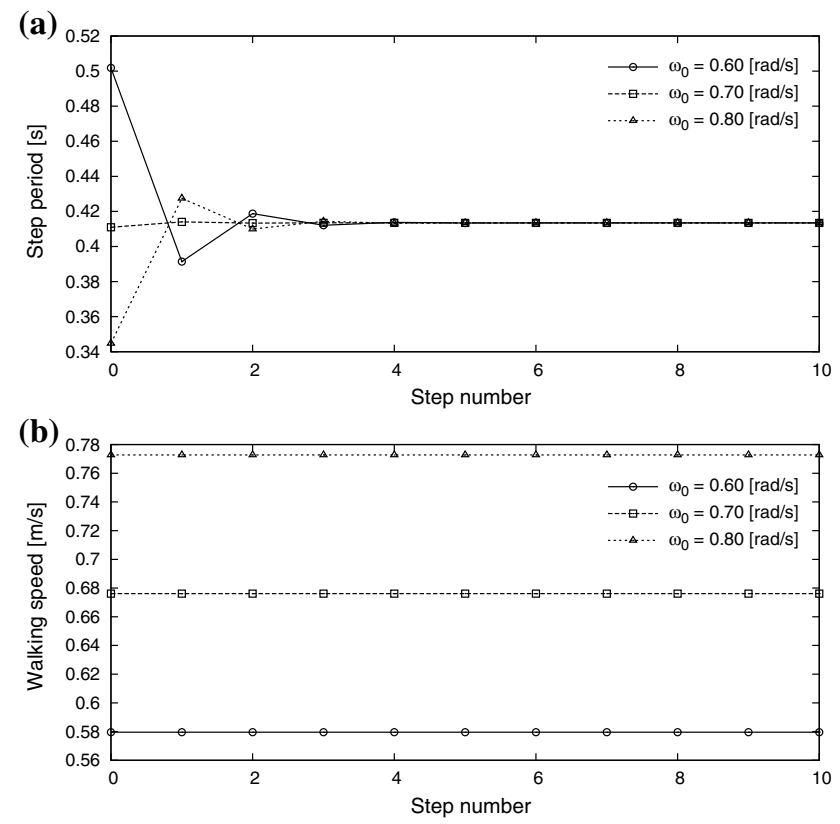

Fig. 4 Evolutions of gait descriptors for three values of initial angular velocities where $T_{\text {set }}=0.30 \mathrm{~s}$ and $\mu_{0}=0$. a Step period, b walking speed

friction enables the walker to increase the forward momentum and to thrust against the floor. The step length is also increased by the effect of sliding.

Figure 6 shows the evolutions of the gait descriptors for three values of $\omega_{0}$ with respect to the step number. Here, (a) is the step period, and (b) is the walking speed. We can confirm that the step period and the walking speed monotonically converge to the steady unique values and that the walker therefore generates a stable 1-period walking gait while sliding. Figure 7 plots the evolutions of the state errors at immediately after impact to check the convergence property in detail. The initial angular velocity is set to be $0.1 \mathrm{rad} / \mathrm{s}$ larger than the steady value. Here, the norm of the velocity vector is defined as $\left\|\dot{\boldsymbol{q}}_{(i)}^{+}-\dot{\boldsymbol{q}}_{\mathrm{eq}}^{+}\right\|$where $\dot{\boldsymbol{q}}_{(i)}^{+} \in \mathbb{R}^{4}$ is the velocity vector at immediately after the $(i)$ th impact and $\dot{\boldsymbol{q}}_{\text {eq }}^{+} \in \mathbb{R}^{4}$ is the steady one (equilibrium point). The errors $\Delta \dot{x}_{(i)}^{+}$and $\Delta \dot{\theta}_{1(i)}^{+}$are also defined as

$\Delta \dot{x}_{(i)}^{+}:=\dot{x}_{(i)}^{+}-\dot{x}_{\mathrm{eq}}^{+}, \quad \Delta \dot{\theta}_{1(i)}^{+}:=\dot{\theta}_{1(i)}^{+}-\dot{\theta}_{1 \mathrm{eq}}^{+}$.

These are the first and the third components of $\dot{\boldsymbol{q}}_{(i)}^{+}-\dot{\boldsymbol{q}}_{\mathrm{eq}}^{+}$and should be plotted to check the sign of the state errors. We can see that the velocity vector norm monotonically converges to zero because it is always positive. The convergence rate, however, significantly changes at the first impact and after that it decreases at a slow speed. $\Delta \dot{x}_{(i)}^{+}$also exhibits the similar property but is always negative. $\Delta \dot{\theta}_{1(i)}^{+}$is positive at the 0 th impact but changes to negative after the first impact. After that, it also converges to zero at a slow speed. In all the cases, the convergence property after the first impact exhibits the 

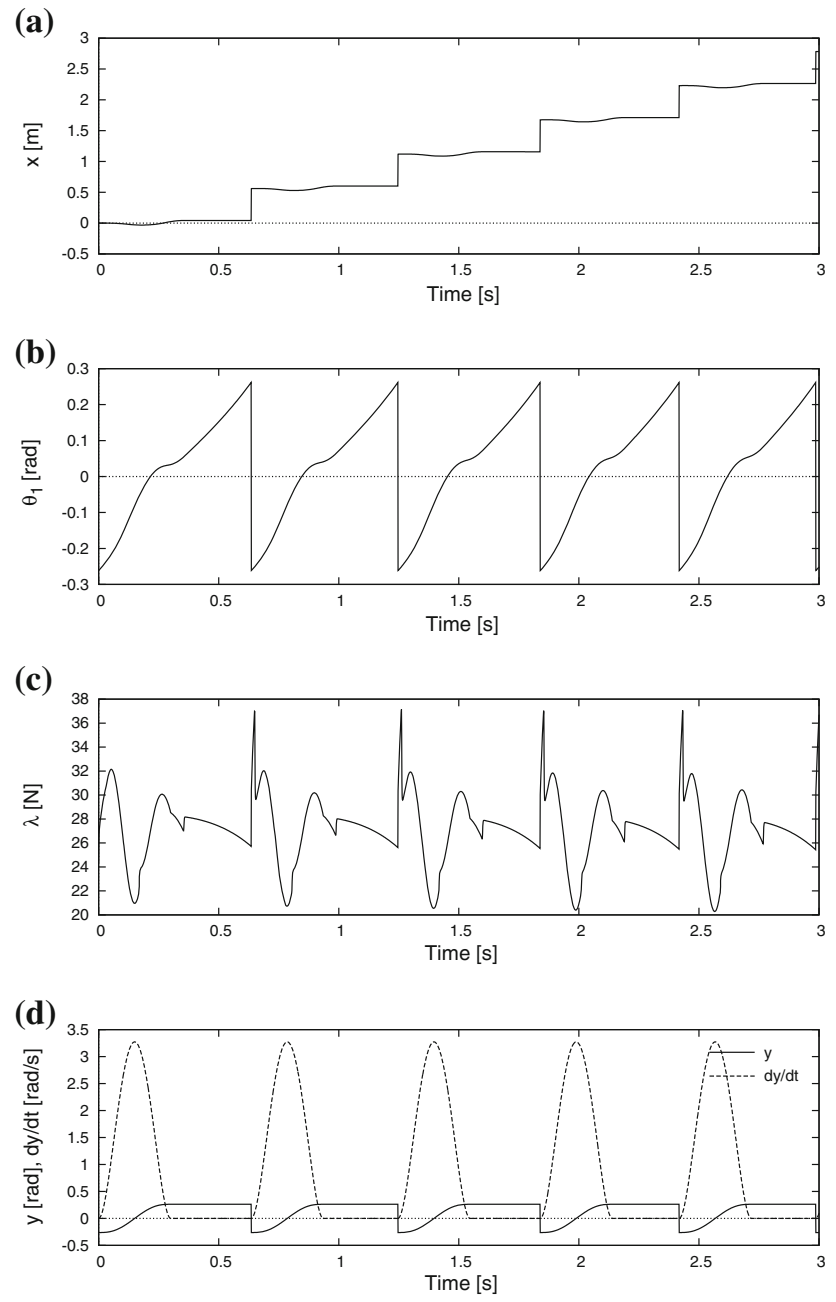

Fig. 5 Simulation results of dynamic walking on slippery surface where $T_{\text {set }}=0.30 \mathrm{~s}$ and $\mu_{0}=0.4$. a $x$. b $\theta_{1}$. c $\lambda$. d $y$ and $\dot{y}$

speed mode; the errors converges to zero at a positive constant rate $[1-3,6]$. The significant change between the 0 th and the first impacts was caused by setting $\dot{x}(0)=\dot{x}_{(0)}^{+}=0 \mathrm{~m} / \mathrm{s}$. The sliding dynamics begins to play in the discrete behavior after the first impact.

\subsection{Gait properties}

Before gait analysis, let us define the gait descriptors for evaluating the efficiency of the generated walking gaits.

The average walking speed, $V(\mathrm{~m} / \mathrm{s})$, is defined as

$V:=\frac{\Delta x}{T}$

Here, $T(\mathrm{~s})$ is the step period and $\Delta x(\mathrm{~m})$ is the step length. $\Delta x$ is defined as the change in $x$ from an instant immediately after impact to the next, i.e. is defined as
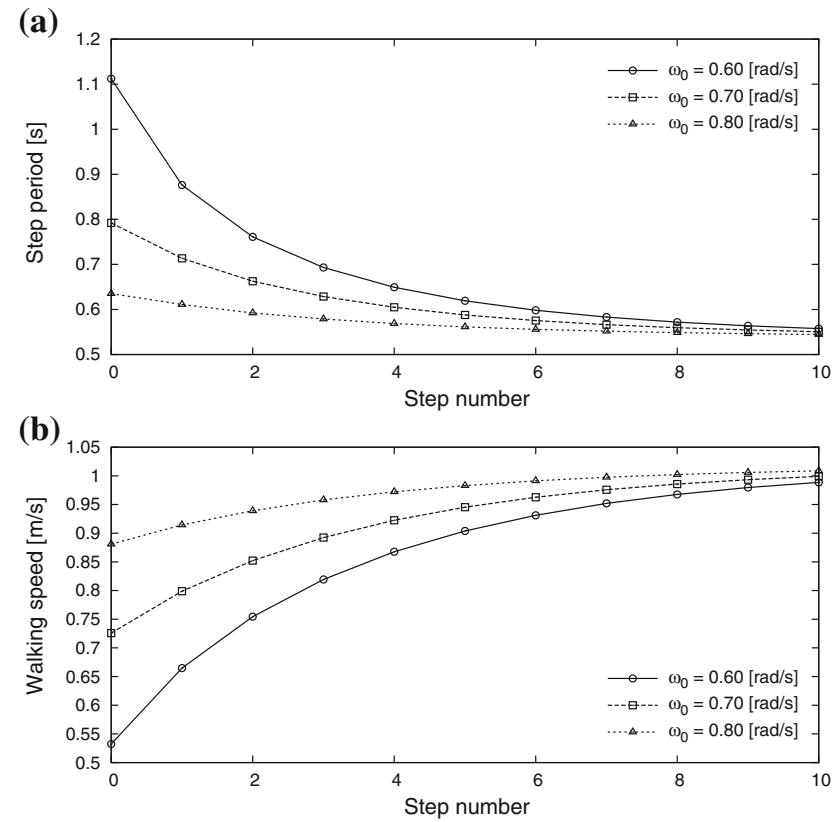

Fig. 6 Evolutions of gait descriptors for three values of initial angular velocities where $T_{\mathrm{set}}=0.30 \mathrm{~s}$ and $\mu_{0}=0.4$. a Step period, $\mathbf{b}$ walking speed

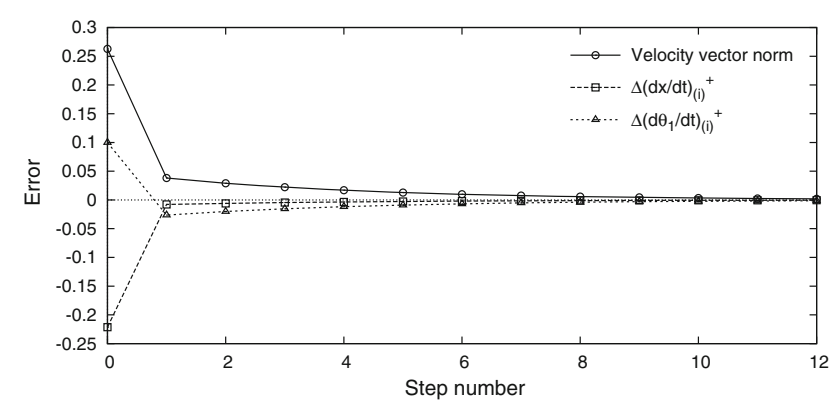

Fig. 7 Evolutions of velocity vector norm and state errors immediately after impact where $T_{\text {set }}=0.30 \mathrm{~s}$ and $\mu_{0}=0.4$

$\Delta x:=\int_{0^{+}}^{T^{-}} \dot{x} \mathrm{~d} t+2 l \sin \frac{\alpha}{2}$.

The energy efficiency is evaluated in terms of the specific resistance (SR) which is a dimensionless quantity and is defined as

$\mathrm{SR}:=\frac{p}{m_{t} g V}$.

Here, $p(\mathrm{~J} / \mathrm{s})$ is the average input power defined by

$p:=\frac{1}{T} \int_{0^{+}}^{T^{-}}|\dot{y} u| \mathrm{d} t=\frac{1}{T} \int_{0^{+}}^{T_{\text {set }}}|\dot{y} u| \mathrm{d} t$.

We performed numerical simulations by taking the following procedures. 

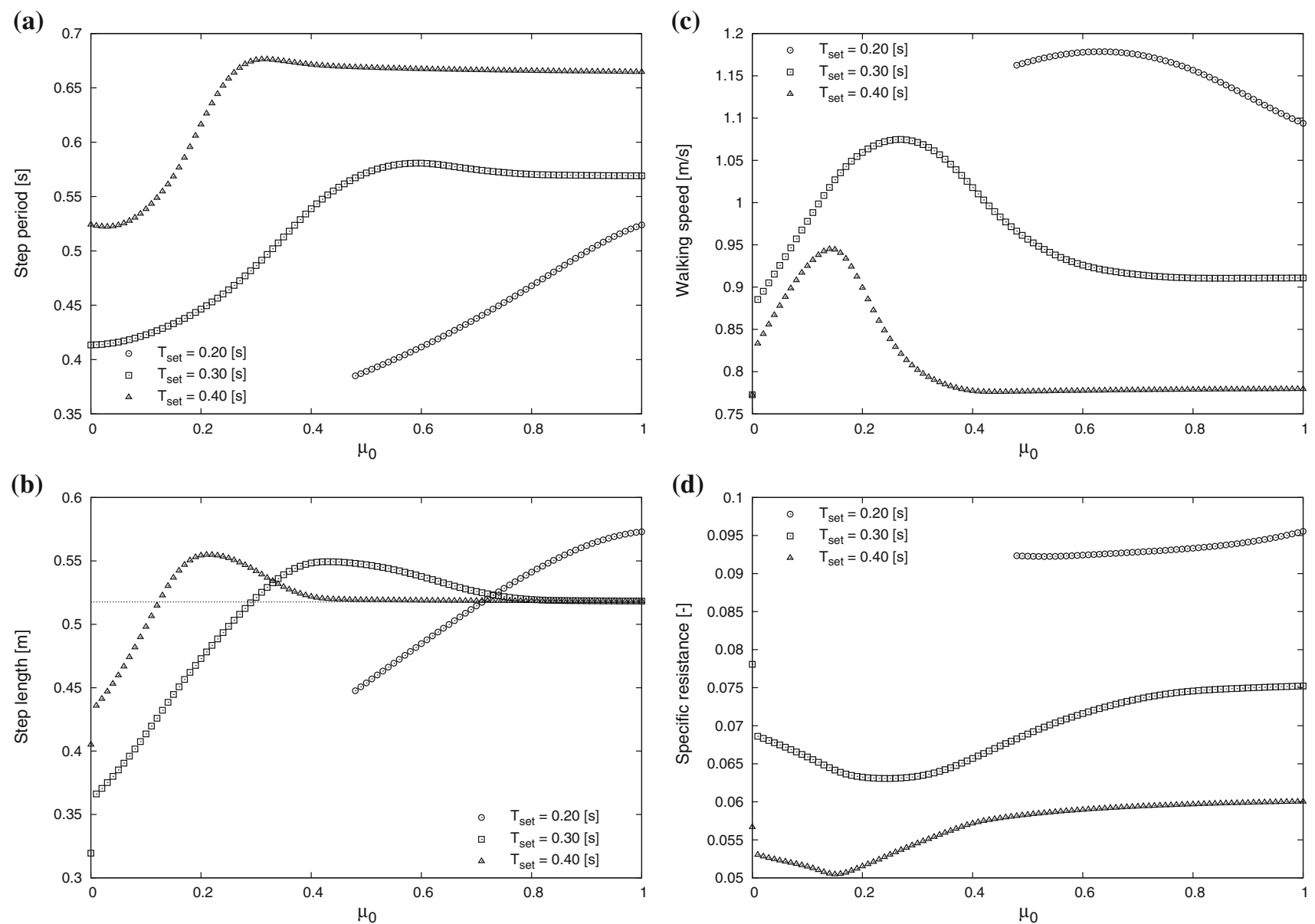

Fig. 8 Gait descriptors for three values of $T_{\text {set }}$ with respect to $\mu_{0}$. a Step period, $\mathbf{b}$ step length, $\mathbf{c}$ walking speed, $\mathbf{d}$ specific resistance

(P1a) Set $\mu_{0}$ to zero.

(P2a) Set the initial conditions to those in Eq. (33) where $\omega_{0}=0.80 \mathrm{rad} / \mathrm{s}$.

(P3a) Run the walking simulation for over $100 \mathrm{~s}$, and save the gait descriptors and the steady state variables immediately after impact.

(P4a) Increase $\mu_{0}$ by 0.01 and rerun the walking simulation by using the state variables saved in (P3a) as the new initial conditions.

(P5a) Repeat from (P3a) until $\mu_{0}=1.0$.

Figure 8 shows the gait descriptors for three values of $T_{\text {set }}$ with respect to $\mu_{0}$. Here, (a) is the step period $T$, (b) the step length $\Delta x$, (c) the walking speed $V$, and (d) the SR. In the cases where $T_{\text {set }}=0.3$ and $0.4 \mathrm{~s}$, stable gaits were generated for all $\mu_{0}$. Where $T_{\text {set }}=0.2 \mathrm{~s}$, stable gaits could not be generated with small values of $\mu_{0}$ because the ground reaction force, $\lambda$, became negative during the stance phases. The small $T_{\text {set }}$ generates a great deal of joint torque, and this induces a substantial change in vertical acceleration.
Except the step period, there are significant differences between the gait descriptors where $\mu_{0}=0$ and those where $\mu_{0}=0.01$. As previously discussed, the gait descriptors where $\mu_{0}=0$ is uniquely determined according to the initial condition of walking. Therefore, the gait efficiency in this case can be unlimitedly improved by increasing the initial angular velocity. The analysis results where $\mu_{0}>0$ suggest that the generated walking motion converges to a unique limit cycle regardless of the initial angular velocity if there is the slightest effect of sliding friction.

From Fig. 8b, we can see that the step lengths in the cases where $T_{\text {set }}=0.30$ and 0.40 s converge to the steady ones and that there are the ranges of $\mu_{0}$ where the step lengths are more than the steady ones. In these ranges, the walker slides forward during the stance phases and the step length becomes longer as shown in Fig. 5a. As $\mu_{0}$ increases more, however, the motion during the stance phases becomes equivalent to that in the absence of sliding due to big friction. The first term of the right-hand side in Eq. (34) therefore converges to zero. To confirm this, we plotted 
$2 l \sin \frac{\alpha}{2}=0.517638 \mathrm{~m}$

in the figure. Also the step length where $T_{\text {set }}=0.20$ would converge to this value if $\mu_{0}$ increases more.

Figure $8 \mathrm{c}, \mathrm{d}$ show that there is a unique $\mu_{0}$ that maximizes the walking speed or minimizes the SR in each case. The walking speed is maximized when the contact point, $x$, slides forward well during the stance phases and the step period is reasonably short. The values of $\mu_{0}$ are close to each other but are different. We must conclude that the optimal solution for $\mu_{0}$ differs according to the criterion.

\section{Passive dynamic walking on slippery slope}

\subsection{Modeling}

In this section, we also use the same walking model but without using the torso as shown in Fig. 9. By setting the control input to zero, we can divide the torso dynamics from the passive RW. The equation of motion is identical to Eq. (19) but the Jacobian matrices, $\boldsymbol{J}$ and $\boldsymbol{J}_{\mu}$, must be accordingly replaced. Let $\phi(\mathrm{rad})$ be the slope angle. The holonomic constraint condition for sliding contact on the slope is then specified as

$\dot{z}=-\dot{x} \tan \phi$,

and the Jacobian becomes

$\boldsymbol{J} \dot{\boldsymbol{q}}=0, \quad \boldsymbol{J}:=\left[\begin{array}{llll}\tan \phi & 1 & 0 & 0\end{array}\right]$.

The component of the ground reaction force normal to the slope is $\lambda / \cos \phi$ and the sliding friction force tangent to the slope is $\mu \lambda / \cos \phi$. Note that the coefficient of sliding

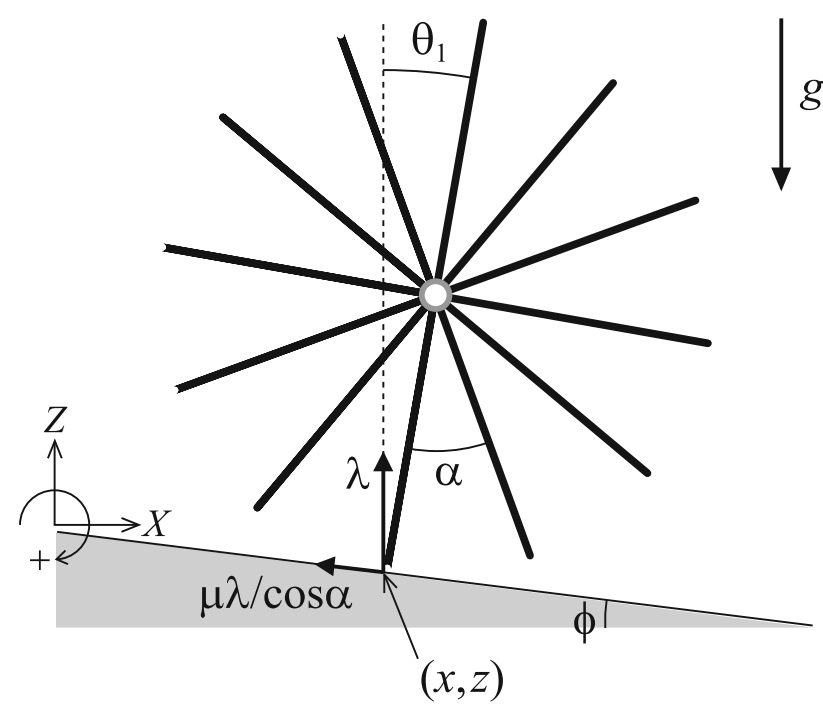

Fig. 9 Model of passive rimless wheel on slippery slope friction, $\mu$, should be reformed taking the tangent velocity of the contact point into account, that is,

$\mu=-\mu_{0} \tanh \left(\frac{c \dot{x}}{\cos \phi}\right)$.

The sliding friction term then becomes

$\left[\begin{array}{c}\cos \phi \\ -\sin \phi \\ 0 \\ 0\end{array}\right] \frac{\mu \lambda}{\cos \phi}=\left[\begin{array}{c}\mu \\ -\mu \tan \phi \\ 0 \\ 0\end{array}\right] \lambda=J_{\mu}^{\mathrm{T}} \lambda$.

Also at impact phase, the holonomic constraint condition must be modified according to the slope angle. Let $(\bar{x}, \bar{z})$ be the position of the fore leg-end at impact. Its time-derivative immediately after impact then becomes

$\frac{\mathrm{d}}{\mathrm{d} t}\left[\begin{array}{c}\bar{x} \\ \bar{z}\end{array}\right]^{+}=\left[\begin{array}{c}\dot{x}+l \dot{\theta}_{1} \cos \theta_{1}^{-}-l \dot{\theta}_{1} \cos \left(\alpha-\theta_{1}^{-}\right) \\ \dot{z}-l \dot{\theta}_{1} \sin \theta_{1}^{-}-l \dot{\theta}_{1} \sin \left(\alpha-\theta_{1}^{-}\right)\end{array}\right]^{+}$.

In this case, however, the $\theta_{1}^{-}$is $\phi+\alpha / 2$. The velocity immediately after impact at the fore leg-end should satisfy

$\dot{\bar{z}}^{+}=-\dot{\bar{x}}^{+} \tan \phi$.

Following Eqs. (39) and (40), the holonomic constraint condition for the collision equation becomes

$\boldsymbol{J}_{I}(\boldsymbol{q}) \dot{\boldsymbol{q}}^{+}=0$,

$\boldsymbol{J}_{I}(\boldsymbol{q})^{\mathrm{T}}=\left[\begin{array}{c}\tan \phi \\ 1 \\ l\left(\sin \left(\phi-\theta_{1}^{-}\right)-\sin \left(\phi-\theta_{1}^{-}+\alpha\right)\right) / \cos \phi \\ 0\end{array}\right]$.

After calculating $\dot{\boldsymbol{q}}^{+}$, we must reset the positional vector as follows. The position of the new stance-leg should be reset to

$x^{+}=\bar{x}=x^{-}+2 l \sin \frac{\alpha}{2} \cos \phi$,
$z^{+}=\bar{z}=z^{-}-2 l \sin \frac{\alpha}{2} \sin \phi$,

and the angular position should be

$\theta_{1}^{+}=\theta_{1}^{-}-\alpha=\frac{\alpha}{2}-\phi$.

In this case, the condition for transition to noninstantaneous DLS motion is given by

$m_{t} l^{2}(1-\cos \alpha) \geq 2 I_{1}$.

Here, note that the right-hand side does not contain the inertia moment of the torso. The value of the left-hand side is the same as the previous case, $0.402 \mathrm{~kg} \mathrm{~m}^{2}$, and the righthand side becomes $1.0 \mathrm{~kg} \mathrm{~m}^{2}$. Therefore, also in this case, the above condition is not satisfied and the rear leg should leave the ground immediately after impact. 

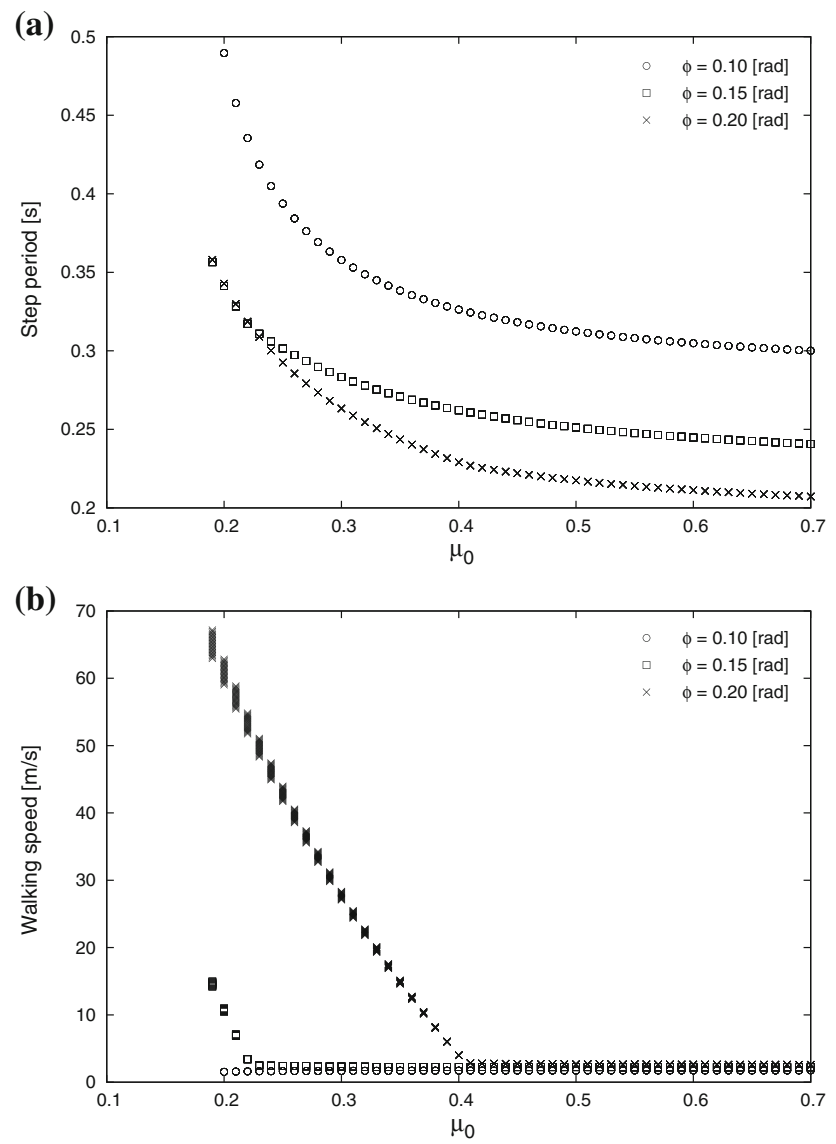

Fig. 10 Gait descriptors for three values of $\phi$ with respect to $\mu_{0}$. a Step period, $\mathbf{b}$ walking speed

\subsection{Gait analysis}

We performed numerical simulations by taking the following procedures.

(P1b) Set $\mu_{0}$ to zero.

(P2b) Set the initial conditions to $\boldsymbol{q}(0)=\mathbf{0}_{4 \times 1}$ and $\dot{\boldsymbol{q}}(0)=$ $\left[\begin{array}{llll}0 & 0 & 0.5 & 0\end{array}\right]^{\mathrm{T}}$.

(P3b) Run the walking simulation for over 100 [s] and save the gait descriptors for 20 steps. Save the steady state variables immediately after the last impact.

(P4b) Increase $\mu_{0}$ by 0.01 and rerun the walking simulation by using the state variables saved in (P3b) as the new initial conditions.

(P5b) Repeat from (P2b) until $\mu_{0}=0.7$.

Figure 10 plots the gait descriptors for three values of $\phi$ with respect to $\mu_{0}$. Here, (a) is the step period and (b) is the walking speed. The step length, $\Delta x$, in this case is defined as the distance traveled along the slope, that is, (a)

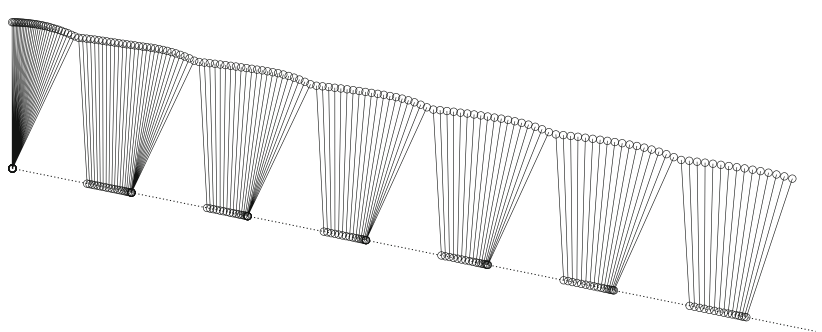

(b)

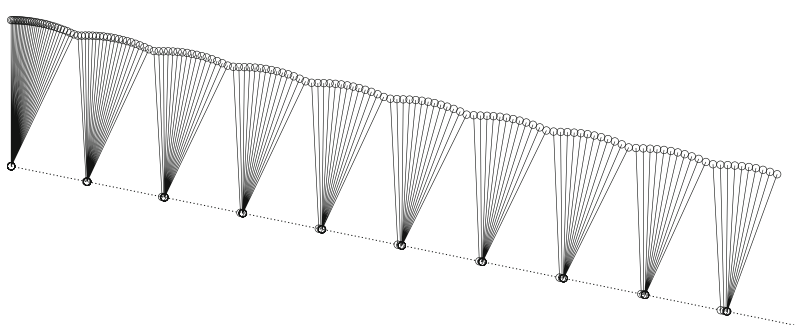

Fig. 11 Stick diagrams for two values of $\mu_{0}$ where $\phi=0.20 \mathrm{rad}$. a $\mu_{0}=0.2$. b $\mu_{0}=0.5$

$\Delta x:=\int_{0^{+}}^{T^{-}} \frac{\dot{x}}{\cos \phi} \mathrm{d} t+2 l \sin \frac{\alpha}{2}$.

The walking speed is then accordingly defined as $V:=$ $\Delta x / T(\mathrm{~m} / \mathrm{s})$. Figure 10a shows that the step period smoothly changes with respect to $\mu_{0}$ and converges to a steady value for a given $\mu_{0}$. Figure 10b shows, however, that the walking speeds where $\phi=0.15$ and 0.20 exhibit non-smooth change rate with respect to $\mu_{0}$ and does not converges in the case with small $\mu_{0}$. Figure 11 plots the stick diagrams for two values of $\mu_{0}$ where $\phi=0.20 \mathrm{rad}$. The RW starts passive dynamic walking from the initial condition of (P2b). The plot where $\mu_{0}=0.2$ strongly supports that the stance leg significantly slides forward during the stance phases, whereas the plot where $\mu_{0}=0.5$ shows that the contact point slightly slides by the effect of the friction force. Figure 12 plots the evolution of $\Delta x$ for four values of $\mu_{0}$ where $\phi=0.20 \mathrm{rad}$. We can see that the step length increases with respect to the step number with small values of $\mu_{0}$. As seen from the above, $\Delta x$ converges to the steady value only if $\mu_{0}$ is sufficiently large. Mathematical investigation of the convergence mechanism is left as a future work.

Figure 13 shows the magnified view of Fig. 10b. Where $\phi=0.10 \mathrm{rad}$, the RW could not generate a stable passivedynamic gait for $\mu_{0}<0.20$. In this case, the slope is too gentle for the RW to overcome the potential barrier at midstance. Figure 14 plots the stick diagram where $\mu_{0}=0.19$ and $\phi=0.10 \mathrm{rad}$. We can see that the RW falls backward after the first impact. Since our simulator cannot detect the impact of the rear leg with the ground, the RW completely falls backward if it fails in overcoming the potential barrier. 


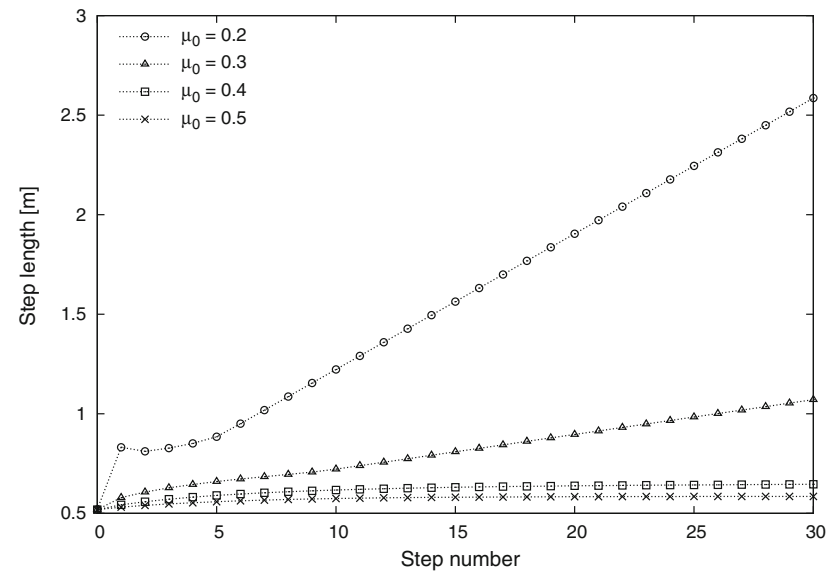

Fig. 12 Evolutions of step length for four values of $\mu_{0}$ where $\phi=$ $0.20 \mathrm{rad}$

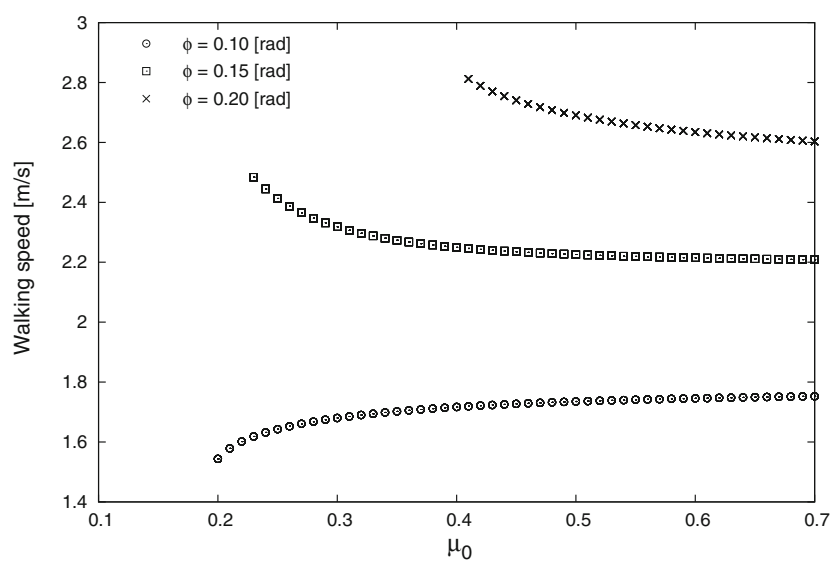

Fig. 13 Magnified view of Fig. 10b

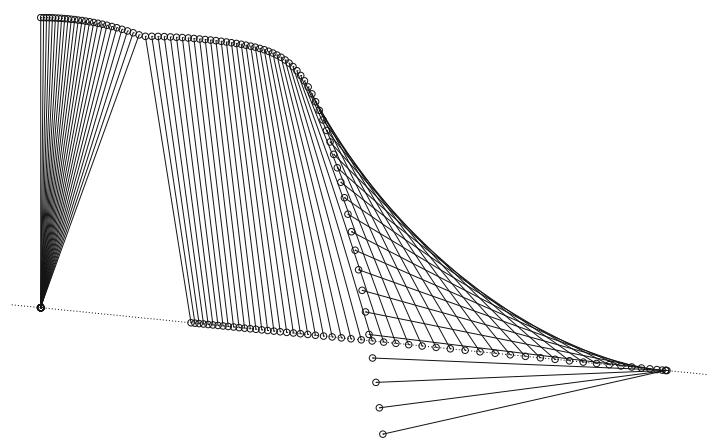

Fig. 14 Stick diagram where $\mu_{0}=0.19$ and $\phi=0.10 \mathrm{rad}$

With large values of $\mu_{0}$, the motion was destabilized soon after the first impact by the effect of chattering around $\dot{x}=0$ due to excessive sliding friction. By setting a small inertia moment, the rotating and sliding motions become highly sensitive to the big friction. This problem can be solved by choosing the inertia moment as a larger value.

\section{Conclusion and future work}

In this paper, we discussed the feasibility of stable limit cycle walking incorporating the effect of sliding friction acting on the stance-leg-end and numerically analyzed the gait properties. Through mathematical analysis, we identified the condition for achieving instantaneous stance-leg exchange. Numerical simulations showed that active and passive dynamic walking can be achieved on frozen/oily surface in appropriate parameter settings and that the friction effect makes the walking gait unique. In passive dynamic walking, however, the generated gait gently diverged where $\mu_{0}$ is small and $\phi$ is large. The convergence mechanism is unexplained at present and more investigations are necessary.

In the future, we should discuss more realistic friction model taking static friction into account such as the LuGre model [11] and extend the mathematical analysis to various walking models. Application to stabilizing control and efficient gait generation taking advantage of frictional effect is also left as a future work.

Acknowledgments This research was partially supported by a Grantin-Aid for Scientific Research, (C) No. 24560542, provided by the Japan Society for the Promotion of Science (JSPS).

\section{References}

1. McGeer T (1990) Passive dynamic walking. Int J Robot Res $9(2): 62-82$

2. Coleman MJ, Chatterjee A, Ruina A (1997) Motions of a rimless spoked wheel: a simple three-dimensional system with impacts. Dyn Stab Syst 12(3):139-159

3. Asano F (2012) Stability principle underlying passive dynamic walking of rimless wheel. In: Proceedings of the IEEE international conference on control applications, pp 1039-1044

4. Bourgeot J-M, Canudas-de-Wit C, Brogliato B (2005) Impact shaping for double support walk: from the rocking block to the biped robot. In: Proceedings of the 8th conference conference on climbing and walking robots and the support technologies for mobile robots, pp 509-516

5. Font-Llagunes JM, Kövecses J (2009) Dynamics and energetics of a class of bipedal walking systems. Mech Mach Theory 44(11):1999-2019

6. Asano F, Xiao X (2012) Output deadbeat control approaches to fast convergent gait generation of underactuated spoked walker. In: Proceedings of the IEEE/SICE international symposium on system Integration (SII), pp 265-270

7. Asano F, Kawamoto J (2014) Modeling and analysis of passive viscoelastic-legged rimless wheel that generates measurable period of double-limb support. Multibody Syst Dyn 31(2):111-126

8. Asano F, Ohshima M (2012) Emergence of measurable period of double-limb support in limit cycle walking with knees. In: Proceedings of the IEEE international conference on control applications, pp 1045-1050

9. Bowden FP (1953) Friction on snow and ice. Proc R Soc Lond A 217(1131):462-478

10. Kuroiwa D (1977) The kinetic friction on snow and ice. J Graciol 19(81):141-152

11. Åström KJ, Canudas-de-Wit C (2008) Revisiting the LuGre friction model. IEEE Control Syst Mag 28(6):101-114 